\title{
Vernier-cascade silicon photonic label-free biosensor with very large sensitivity and low-cost interrogation
}

\author{
Tom Claes ${ }^{a, b}$, Wim Bogaerts ${ }^{a, b}$ and Peter Bienstman ${ }^{a, b}$ \\ ${ }^{a}$ Photonics Research Group, Department of Information Technology \\ Ghent University - IMEC, Sint-Pietersnieuwstraat 41, 9000 Gent, Belgium \\ ${ }^{b}$ Center for Nano- and Biophotonics (NB-Photonics), Ghent University
}

\begin{abstract}
Recently, cheap silicon-on-insulator label-free ring resonator biosensors have been demonstrated that allow fast and accurate quantitative detection of biologically relevant molecules for applications in medical diagnostics and drug development. However, a further improvement of their detection limit is limited by their small sensitivity and an expensive tunable laser is typically required to resolve the sharp resonances for wavelength interrogation. Therefore, we experimentally investigated the use of a Vernier-cascade sensor that achieves a sensitivity (thousands of $\mathrm{nm} / \mathrm{RIU}$ ) that is an order of magnitude larger than that of a ring resonator sensor (approx. 100 $\mathrm{nm} / \mathrm{RIU}$ ), while still maintaining sharp spectral features that allow precise monitoring of spectral shifts with data-fitting. Moreover we prove that it's also possible to accurately interrogate the sensor with a low-cost broadband light source by integrating it with an arrayed waveguide grating spectral filter that divides the sensor's transmission spectrum in multiple wavelength channels and transmits them to spatially separated output ports. Experiments show that this sensor can monitor refractive index changes of watery solutions in real-time with a detection limit $\left(1.6 \cdot 10^{-5} R I U\right)$ competitive with more expensive interrogation schemes, indicating its applicability in low-cost label-free biosensing.
\end{abstract}

Keywords: silicon, label-free biosensing, ring resonator, Vernier, low-cost interrogation

\section{INTRODUCTION}

Label-free photonic biosensors can perform sensitive and quantitative multiparameter measurements on biological systems and can therefore contribute to major advances in medical analyses, food quality control, drug development and environmental monitoring. Additionally they offer the prospect of being incorporated in laboratorieson-a-chip that are capable of doing measurements at the point-of-care at an affordable cost. ${ }^{1,2}$

A crucial component in most of these photonic biosensors is a transducer that can transform a refractive index change in its environment to a measurable change in its optical transmission. Silicon-on-insulator (SOI) is a material system with many assets for such transducers. First, it has a high refractive index contrast permitting very compact sensors of which many can be incorporated on a single chip, enabling multiplexed sensing. Second, silicon-on-insulator photonic chips can be made with CMOS-compatible process steps, allowing for a strong reduction of the chip cost by high volume fabrication. These sensor chips can therefore be disposable, meaning that the chip is only used once, avoiding complex cleaning of the sensor surface after use. Typically, a spectral shift of the transmission spectrum of the transducer is used to quantify the measured refractive index change, because this method can be extended to the parallel read-out of multiple sensors in a sensor matrix.

Recently, we and other research groups have demonstrated SOI ring resonator sensor matrices capable of realtime multiparameter measurements. ${ }^{3-5}$ Ring resonators achieve good detection limits (down to $7.610^{-7} \mathrm{RIU}$, ref. $^{4}$ ) because they have very sharp resonances of which spectral shifts can be monitored accurately. However, this spectral shift is typically small (in the order of a hundred nm/RIU), preventing further improvement of the detection limit. Additionally, these sharp resonances require an expensive tunable laser to be resolved, significantly increasing the cost of a device.

Send correspondence to tom.claes@intec.ugent.be 
Therefore, we propose to use a Vernier-cascade sensor 6,7 that consists of two cascaded ring resonators with different roundtrips and that can be made more than an order of magnitude more sensitive than a ring resonator sensor (sensitivities of several thousands of $\mathrm{nm} / \mathrm{RIU}$ ). Moreover, $\mathrm{in}^{6}$ we demonstrated that this sensor can be designed to have a periodic envelope signal superposed on very sharp constituent peaks, thus combining a large sensitivity with accurately resolvable spectral features. The sharp constituent peaks can be resolved with a tunable laser, to ultimately improve the detection limit compared to ring resonator sensors. Alternatively, the envelope signal can directly be resolved with a low-resolution integrated spectral filter, allowing much cheaper read-out with a broadband light source.

First, this conference paper reports formulas that accurately describe the transmission spectrum of the sensor, and presents a fitting procedure based on these formulas to accurately monitor spectral shifts of the sensor's transmission spectrum. Second, it discusses experimental results of a compact implementation of a Verniercascade sensor in silicon-on-insulator interrogated with a tunable laser. Third, it presents the first integration of this sensor with an arrayed waveguide grating spectral filter and demonstrates interrogation with a low-cost broadband light source.

\section{THEORETICAL ANALYSIS OF THE SENSOR}

The Vernier-scale is a method to enhance the accuracy of measurement instruments. It consists of two scales with different periods, of which one slides along the other one. The overlap between lines on the two scales is used to perform the measurement. It is commonly used in calipers and barometers, and it has also been applied in photonic devices. 8,9

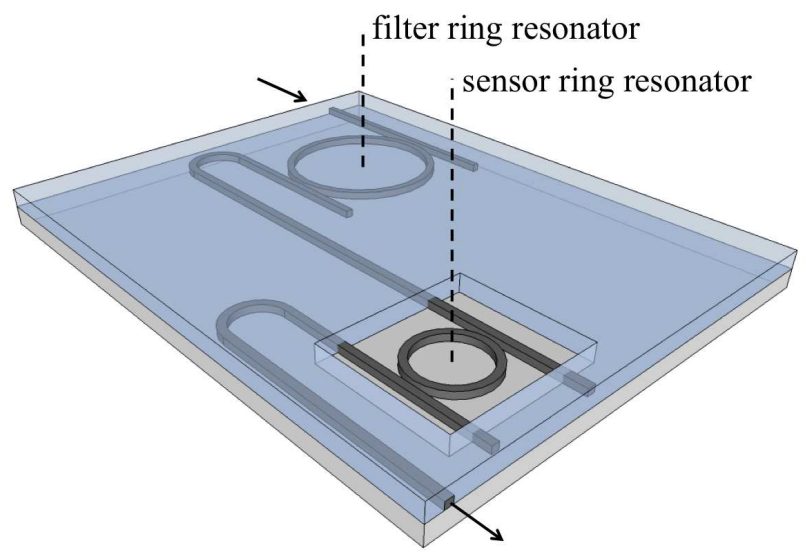

Figure 1. Illustration of the concept of the photonic sensor consisting of two cascaded ring resonators. Two ring resonators with different optical roundtrip lengths are cascaded. The complete chip is covered with a thick cladding, with only an opening for one of the two resonators. This sensor ring resonator will be exposed to refractive index changes in its environment, while the other resonator, the filter ring resonator, is shielded from these refractive index changes by the cladding.

In Fig. 1 it is illustrated how this concept can be applied to a ring resonator sensor. Two ring resonators with different optical roundtrip lengths are cascaded, so that the drop signal of the first ring resonator serves as the input of the second. Each individual ring resonator has a comb-like transmission spectrum with peaks at its resonance wavelengths. The spectral distance between these peaks, the free spectral range, is inversely proportional to the optical roundtrip of the resonator, so that each resonator in the cascade will have a different free spectral range. As the transmission spectrum of the cascade of the two ring resonators is the product of the transmission spectra of the individual resonators, it will only exhibit peaks at wavelengths for which two resonance peaks of the respective ring resonators (partially) overlap, and the height of each of these peaks will be determined by the amount of overlap. 
The complete chip is covered with a thick cladding, with only an opening for one of the two resonators. This sensor ring resonator will act as the sliding part of the Vernier-scale, as its evanescent field can interact with the refractive index in the environment of the sensor, where a change will cause a shift of the resonance wavelengths. The other resonator, the filter ring resonator, is shielded from these refractive index changes by the cladding and will act as the fixed part of the Vernier-scale. The cascade of both resonators can be designed such that a small shift of the resonance wavelengths of the sensor ring resonator will result in a much larger shift of the transmission spectrum of the cascade.
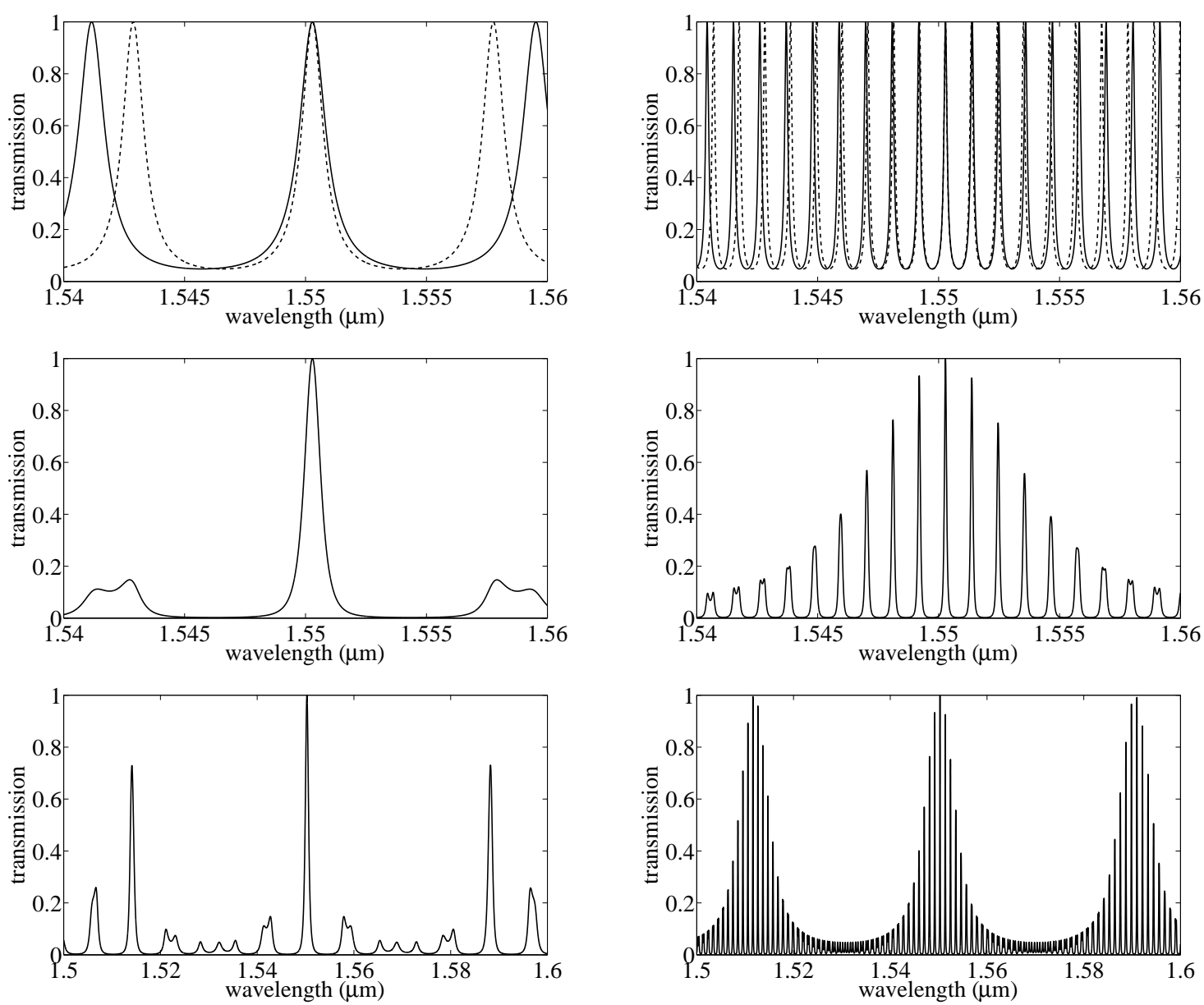

Figure 2. Calculated transmission spectra that illustrate the operation of the cascade. The graphs on the left side illustrate a first regime that occurs when the free spectral range difference between the two resonators is large compared to the full-width at half-maximum of the resonance peaks of the individual resonators. The graphs on the right side illustrate a second regime that occurs when the free spectral range difference between the two resonators is small compared to the full-width at half-maximum of the resonance peaks of the individual resonators. Top: transmission spectra of the individual filter ring resonator (dashed line) and sensor ring resonator (normal line). Middle: transmission spectra of the cascade of these two resonators in the same wavelength range as the top image, illustrating only one clearly visible transmitted peak in the first regime (left), while in the second regime (right) an envelope signal is superposed on the constituent peaks. Bottom: transmission spectra of the cascade in a larger wavelength range.

\section{$\mathrm{In}^{6}$ we reported that two regimes can be identified:}

The first regime is illustrated on the left side of Fig. 2 and occurs when the free spectral range difference between the two resonators in the cascade is large compared to the full-width at half-maximum of the resonance peaks of the individual resonators. The transmission spectrum of the cascade will then typically exhibit isolated peaks, of which the neighboring peaks are inhibited. The sensor will in this regime behave as a discrete sensor, 
of which the transmission peak will hop from one filter ring resonance wavelength to another for a changing refractive index. The smallest detectable shift of the transmission spectrum of this sensor is equal to the free spectral range of the filter ring resonator, which forms a limitation to the detection limit of the sensor.

A second regime occurs however when the free spectral range difference between the two resonators in the cascade is small compared to the full-width at half-maximum of the resonance peaks of the individual resonators. On the right side of Fig. 2 it is illustrated that in this regime a periodic envelope signal is superposed on the constituent transmission peaks. If we do not take dispersion into account, the envelope period is given by

$$
\frac{f s r_{\text {sensor }} \cdot f s r_{\text {filter }}}{\left|f s r_{\text {sensor }}-f s r_{\text {filter }}\right|}
$$

where $f s r_{\text {sensor }}$ and $f s r_{\text {filter }}$ are the free spectral range values of the corresponding individual resonators. Note that in practice the envelope period can not be chosen larger than the available wavelength range of the measurement equipment, so that the second regime typically requires that the cascade consists of resonators with very large roundtrips. In the remainder of this article, we will work with sensors that work in this regime.

$\operatorname{In}^{6}$ we showed that the constituent peaks can be described by

$$
T_{\text {constituent }}(\lambda)=\frac{t_{\text {max }, \text { filter }} \frac{f w h m_{\text {filter }}^{2}}{4}}{\frac{f w h m_{\text {filter }}^{2}}{4}+\left(\lambda-\lambda_{0}-\frac{\Delta \lambda}{2}\right)^{2}} \cdot \frac{t_{\text {max }, \text { sensor }} \frac{f w h m_{\text {sensor }}^{2}}{4}}{\frac{f w h m_{\text {sensor }}^{2}}{4}+\left(\lambda-\lambda_{0}+\frac{\Delta \lambda}{2}\right)^{2}}
$$

where $t_{\max }$ and $f w h m$ are respectively the transmission at resonance and the full-width at half-maximum of the corresponding individual ring resonator, and where $\lambda_{0}$ and $\Delta \lambda$ are respectively the mean of and the difference between the two resonance wavelengths under consideration from both combs.

$\mathrm{In}^{6}$ it is also proven that the envelope peak in the transmission of the cascade formed by the maxima of the constituent peaks is in good approximation given by

$$
T_{\text {envelope }}(\lambda)=\left(\frac{\sqrt{t_{\text {max }, \text { filter }} t_{\text {max }, \text { sensor }}}\left(\frac{F W H M}{2}\right)^{2}}{\left(\frac{F W H M}{2}\right)^{2}+\left(\lambda-\lambda_{\text {central }}\right)^{2}}\right)^{2}
$$

with

$$
F W H M=2 \cdot \frac{f w h m \cdot \min \left(f s r_{\text {sensor }}, f s r_{\text {filter }}\right)}{\left|f s r_{\text {filter }}-f s r_{\text {sensor }}\right|}
$$

Here $\lambda_{\text {central }}$ is defined as the central wavelength of the envelope peak. Eq. (3) shows that the envelope signal forms a peak described by the square of a Lorentzian function with full-width at half-maximum $F W H M$.

A change of the refractive index in the evanescent field of the sensor ring resonator will cause a shift of the resonance peaks in its transmission spectrum, which will be translated in a much larger shift of the central wavelength of the envelope peak in the transmission spectrum of the cascade. The sensitivity of the cascaded ring resonator sensor is given by

$$
\frac{\partial \lambda_{\text {central }}}{\partial n_{\text {env }}}=\frac{f s r_{\text {filter }}}{f s r_{\text {filter }}-f s r_{\text {sensor }}} \frac{\frac{\partial n_{\text {eff,sensor }}}{\partial n_{\text {env }}} \lambda}{n_{g, \text { sensor }}}
$$

with $\frac{\partial n_{\text {eff.sensor }}}{\partial n_{\text {env }}}$ the change of the effective index of the sensor ring resonator waveguide due to a change of the refractive index in the environment of the sensor and $n_{g \text {,sensor }}$ the group index of the sensor ring resonator waveguide. The sensitivity of the cascaded ring resonator sensor is enhanced with a factor $\frac{f s r_{f i l t e r}}{f s r_{f i l t e r}-f s r_{s e n s o r}}$ compared to the sensitivity of a single ring resonator sensor. As already stated earlier, in practice the period of the envelope signal of the cascade cannot be chosen larger than the available wavelength range of the measurement equipment. For a given envelope period, Eq. (1) and (5) show that the sensitivity is in good approximation proportional to the optical roundtrip length of the resonators in the cascaded. Note that for an increasing refractive index $n_{\text {env }}$ the resonance wavelength of a single ring resonator will always shift to larger wavelengths, while the central wavelength of the envelope peak in the transmission spectrum of the cascade will shift to smaller wavelengths if $f s r_{f i l t e r}<f s r_{\text {sensor }}$ and to larger wavelengths if $f s r_{f i l t e r}>f s r_{\text {sensor }}$. 


\section{EXPERIMENTAL CHARACTERIZATION OF A SILICON VERNIER-CASCADE SENSOR}

A Vernier-cascade sensor was fabricated in silicon-on-insulator with CMOS-compatible 193nm optical lithography and dry etching. ${ }^{10}$ To reduce the device's footprint, the resonator cavities were folded (Fig. 3).

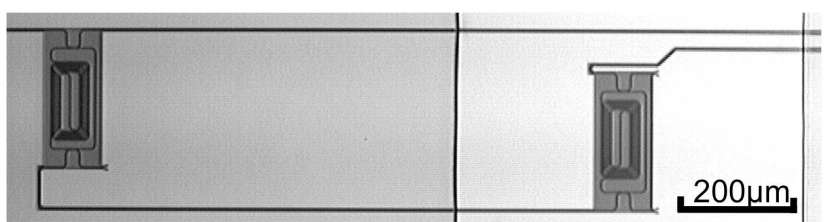

Figure 3. Optical microscope image of the device fabricated in silicon-on-insulator. Two ring resonators with $2.5 \mathrm{~mm}$ physical roundtrip length are cascaded, and their footprint is reduced by folding the cavity. The complete chip was covered with $500 \mathrm{~nm}$ silicon oxide, and an opening was etched to the second ring resonator.

The complete chip was covered with 500nm silicon oxide by plasma deposition and a window was etched to the second resonator in the cascade by consecutive dry and wet etching, so that only the evanescent field of this sensor ring resonator can interact with refractive index changes in the environment of the sensor.

To allow controlled delivery of liquids to the sensor, a microfluidic channel in PDMS with $600 \mu m \times 50 \mu m$ cross section was bonded to the sensor chip. ${ }^{11}$ The liquids were pumped through the channel over the sensor ring resonator with a syringe pump at a $5 \mu \mathrm{L} / \mathrm{min}$ flow rate. The chip was mounted on a temperature-stabilized chuck to avoid drifting of the sensor signal due to temperature variations. A second-order diffractive grating, integrated on the input and output waveguides, is used to couple from a $10 \mu \mathrm{m}$ wide ridge waveguide to a vertically oriented, butt-coupled single-mode fiber. A linear, $150 \mu \mathrm{m}$ long taper is employed as a transition between the ridge waveguide and a $450 \mathrm{~nm}$ wide photonic wire waveguide. ${ }^{12}$ A polarization controller was used to tune the polarization of light from a tunable laser for maximum coupling to the quasi-TE mode of the waveguides, and the optical power transmitted by the sensor was measured with a photodetector.

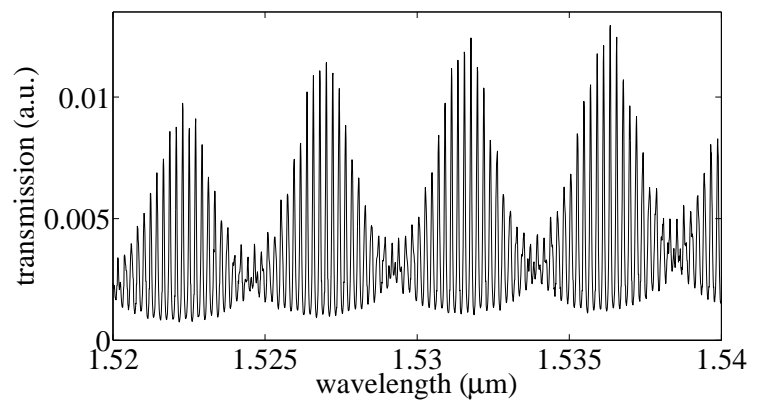

Figure 4. Measured transmission spectrum of the device as deionized water is flowing over the sensor ring resonator. The height of the envelope peaks varies due to the wavelength-dependent coupling efficiency of the grating couplers.

As expected, a periodic envelope signal is superposed on the constituent transmission peaks of the device (Fig. 4). To accurately monitor spectral shifts of the envelope signal, we consecutively fitted formula 2 to the sharp constituent peaks and formula 3 to the maxima of the these constituent peaks to reveal the superposed envelope peak (Fig. 5).

A good measure for the smallest detectable wavelength shift with this method is given by the standard deviation on the fitted central wavelength of the envelope peak. Based on the confidence interval of the fitting parameters returned by our standard fitting software, the smallest detectable wavelength shift was calculated to be $18 \mathrm{pm}$ for the measured spectra of our sensor. Note that this value is an order of magnitude smaller than the distance between the peaks in the spectrum.

To measure the sensitivity of the sensor to changes in the bulk refractive index of its aqueous environment, it was measured how much the envelope peaks in the transmission spectrum shifted when changing between 


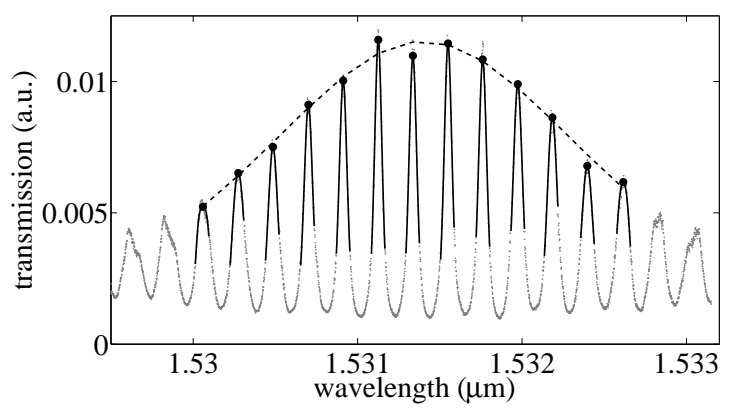

Figure 5. Illustration of the fitting procedure. In grey a measured transmission spectrum of our device is shown. In a first step Eq. (2) is fitted to the highest constituent peaks, shown by the solid lines. Then the analytical maxima of these fits are determined, shown by the dots. In a second step, Eq. (3) is fitted to the envelope signal formed by these maxima, which is shown by the dashed line. The position of the analytical maximum of that last fit is taken as the central wavelength of the envelope peak.

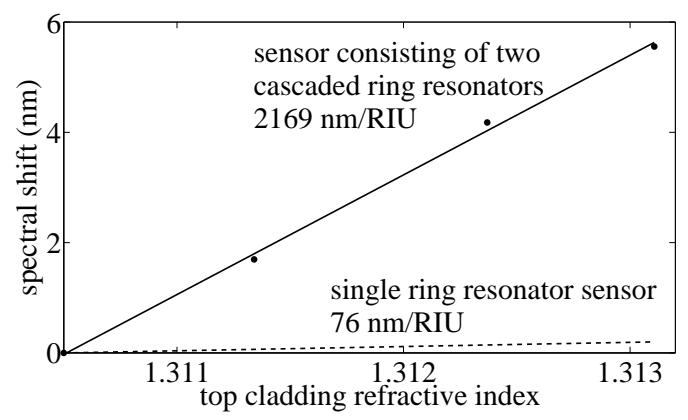

Figure 6. Shift of the transmission spectrum of the sensor consisting of two cascaded ring resonators as a function of the bulk refractive index in its top cladding. The dots show the shift that was measured by changing the flow between deionized water and aqueous solutions of $\mathrm{NaCl}$ with different concentrations, and the solid line represents the linear fit to this experimental data, revealing a sensitivity of $2169 \mathrm{~nm} / \mathrm{RIU}$. For comparison, the dashed line shows the calculated resonance wavelength shift of a single ring resonator.

flowing deionized water and three aqueous solutions of $\mathrm{NaCl}$ with different concentrations. The refractive index of each of these solutions was calculated. ${ }^{13}$ In Fig. 6 the dots indicate the measured shifts as a function of bulk refractive index. A linear function was fitted to the measured shifts, and its slope revealed a sensitivity of $2169 \mathrm{~nm} / \mathrm{RIU}$. This value corresponds well with the theoretical sensitivity of $2085 \mathrm{~nm} / \mathrm{RIU}$ calculated with Eq. (5). The resonance wavelength shift of a single ring resonator comprised of a $450 \mathrm{~nm}$ wide waveguide is calculated to be $76 \mathrm{~nm} / \mathrm{RIU}$, showing the large sensitivity improvement with the presented sensor.

The resulting detection limit of our sensor is equal to the ratio of the smallest detectable wavelength shift and the sensitivity, that is $18 \mathrm{pm} / 2169 \mathrm{~nm} / \mathrm{RIU}=8.310^{-6} \mathrm{RIU}$. Although the detection limit is currently not improved compared to that of a single ring resonator sensor, ${ }^{4}$ we think this first experimental result is promising for future optimized designs regarding the different levels of optimization of single ring resonator sensors and this new sensor.

\section{VERNIER-CASCADE SENSOR WITH INTEGRATED ARRAYED WAVEGUIDE GRATING FOR WAVELENGTH INTERROGATION WITH LOW-COST BROADBAND SOURCE}

To reduce the cost of the sensor interrogation, we integrated a silicon Vernier-cascade sensor with an arrayed waveguide grating (AWG) to divided the sensor's transmission spectrum in multiple wavelength channels and distribute them to spatially separated output ports (Fig. 7). The AWG has 16 channels with $1.6 \mathrm{~nm}$ spacing, so that its bandwidth is larger than the envelope period of the sensor $(13 \mathrm{~nm})$ to avoid it from limiting the sensor's dynamic range. The chosen channel spacing is the result of a trade-off between having a high resolution and 
having enough spectral averaging per channel to be able to disclose the envelope signal directly with a broadband light source. The AWG's footprint is only $750 \mu m \times 350 \mu m$.

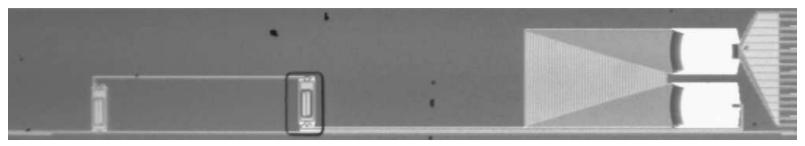

Figure 7. The transmission spectrum of a Vernier-cascade sensor can be measured with a low-cost broadband light source by dividing it into different wavelength channels with an integrated arrayed waveguide grating (AWG) that distributes them to spatially separated output ports that can be monitored in parallel with a camera. The micrograph of the silicon-on-insulator chip shows the compact folded cavities of the ring resonators constituting the Vernier-cascade that is connected to a dense 16-channel AWG.

Light from a low-cost light emitting diode (LED) was coupled to this device and the relative power diffracted to free space by all output gratings, each corresponding with a channel of the AWG, was monitored in parallel using a near-infrared camera with a microscope objective at a distance of several centimeters from the chip. The relative transmission of the AWG-channels directly reveals the envelope signal, as each channel is much broader than the spacing between the constituent peaks (Fig. 8). The performance of this device as transducer

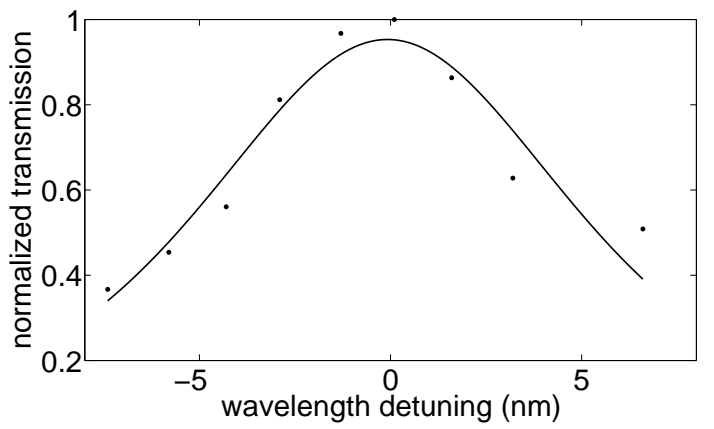

Figure 8. The shape of the envelope peak is clearly visible when the transmission is measured with a broadband light source (dots). A squared Lorentzian function can be satisfactory fitted to this broadband transmission peak (line), allowing accurate determination of the peak position.

for label-free biosensing was characterized by measuring the refractive index change of watery salt solutions in real-time and it appeared that despite the limited spectral resolution of the AWG and corresponding inaccuracy of the peak fitting, the device allows to monitor refractive index changes accurately thanks to the much faster read-out (no need to sweep a laser source) that allows averaging. The detection limit $\left(1.6 \cdot 10^{-5} R I U\right)$ is in the same order as the detection limit of the Vernier-sensor interrogated with a much more expensive tunable laser $\left(8.3 \cdot 10^{-6} R I U\right.$, section 3$)$.

\section{CONCLUSIONS}

A Vernier-cascade sensor is more than an order of magnitude more sensitive than a ring resonator sensor $(2000 \mathrm{~nm} / \mathrm{RIU}$ instead of $100 \mathrm{~nm} / \mathrm{RIU})$ and has a transmission spectrum that consists of accurately resolvable sharp peaks, giving it the prospect of improving the detection limit of current silicon-on-insulator label-free biosensors. Moreover, we proved it can be integrated with an arrayed waveguide grating to allow wavelength interrogation with a low-cost broadband light source without significant reduction of its performance, indicating its applicability in low-cost label-free biosensing

\section{Acknowledgements}

The authors thank ePIXfab (www.epixfab.eu) for the fabrication of the device and Steven Verstuyft for help during the final steps of the fabrication. Tom Claes was supported by the Flemish Institute for the Promotion of Innovation through Science and Technology (IWT) with a specialization grant and Wim Bogaerts by the Flemish 
Research Foundation with a postdoctoral fellowship. This work was performed in the context of the European project InTopSens and the Belgian IAP project Photonics@BE.

\section{REFERENCES}

1. X. Fan, I. M. White, S. I. Shopoua, H. Zhu, J. D. Suter, and Y. Sun, "Sensitive optical biosensors for unlabeled targets: A review," Analytica Chimica Acta 620, pp. 8-26, JUL 142008.

2. A. J. Qavi, A. L. Washburn, J.-Y. Byeon, and R. C. Bailey, "Label-free technologies for quantitative multiparameter biological analysis," Anal. Bioanal. Chem. 394, pp. 121-135, May 2009.

3. K. De Vos, J. Girones, T. Claes, Y. De Koninck, S. Popelka, E. Schacht, R. Baets, and P. Bienstman, "Multiplexed antibody detection with an array of silicon-on-insulator microring resonators," IEEE Photon. J. 1, pp. 225-235, Oct. 2009.

4. M. Iqbal, M. A. Gleeson, B. Spaugh, F. Tybor, W. G. Gunn, M. Hochberg, T. Baehr-Jones, R. C. Bailey, and L. C. Gunn, "Label-free biosensor arrays based on silicon ring resonators and high-speed optical scanning instrumentation," IEEE J. Sel. Topics Quantum Electron. 16, pp. 654-661, May-June 2010.

5. D. X. Xu, M. Vachon, A. Densmore, R. Ma, A. Delage, S. Janz, J. Lapointe, Y. Li, G. Lopinski, D. Zhang, Q. Y. Liu, P. Cheben, and J. H. Schmid, "Label-free biosensor array based on silicon-on-insulator ring resonators addressed using a wdm approach," Opt. Lett. 35, pp. 2771-2773, Aug 2010.

6. T. Claes, W. Bogaerts, and P. Bienstman, "Experimental characterization of a silicon photonic biosensor consisting of two cascaded ring resonators based on the vernier-effect and introduction of a curve fitting method for an improved detection limit," Opt. Express 18(22), pp. 22747-22761, 2010.

7. L. Jin, M. Li, and J.-J. He, "Highly-sensitive silicon-on-insulator sensor based on two cascaded micro-ring resonators with vernier effect," Opt. Commun. 284(1), pp. 156 - 159, 2011.

8. B. Liu, A. Shakouri, and J. Bowers, "Wide tunable double ring resonator coupled lasers," IEEE Photonics Technology Letters 14, pp. 600-602, MAY 2002.

9. P. Rabiei and W. Steier, "Tunable polymer double micro-ring filters," IEEE Photonics Technology Letters 15, pp. 1255-1257, SEP 2003.

10. S. K. Selvaraja, P. Jaenen, W. Bogaerts, D. V. Thourhout, P. Dumon, and R. Baets, "Fabrication of photonic wire and crystal circuits in silicon-on-insulator using 193-nm optical lithography," J. Lightw. Technol. 27(18), pp. 4076-4083, 2009.

11. S. Bhattacharya, A. Datta, J. Berg, and S. Gangopadhyay, "Studies on surface wettability of poly(dimethyl) siloxane (pdms) and glass under oxygen-plasma treatment and correlation with bond strength," IEEE Journal of Microelectromechanical Systems 14, pp. 590-597, JUN 2005.

12. W. Bogaerts, D. Taillaert, B. Luyssaert, P. Dumon, J. V. Campenhout, P. Bienstman, D. V. Thourhout, R. Baets, V. Wiaux, and S. Beckx, "Basic structures for photonic integrated circuits in silicon-on-insulator," Opt. Express 12(8), pp. 1583-1591, 2004.

13. H. Su and X. G. Huang, "Fresnel-reflection-based fiber sensor for on-line measurement of solute concentration in solutions," Sens. Actuators, B 126, pp. 579-582, OCT 12007. 